AperTO - Archivio Istituzionale Open Access dell'Università di Torino

\title{
Goal or movement? Action representation within the primary motor cortex
}

\section{This is the author's manuscript}

Original Citation:

Availability:

This version is available http://hdl.handle.net/2318/143461

since

Published version:

DOI:10.1111/ejn.12343

Terms of use:

Open Access

Anyone can freely access the full text of works made available as "Open Access". Works made available under a Creative Commons license can be used according to the terms and conditions of said license. Use of all other works requires consent of the right holder (author or publisher) if not exempted from copyright protection by the applicable law. 


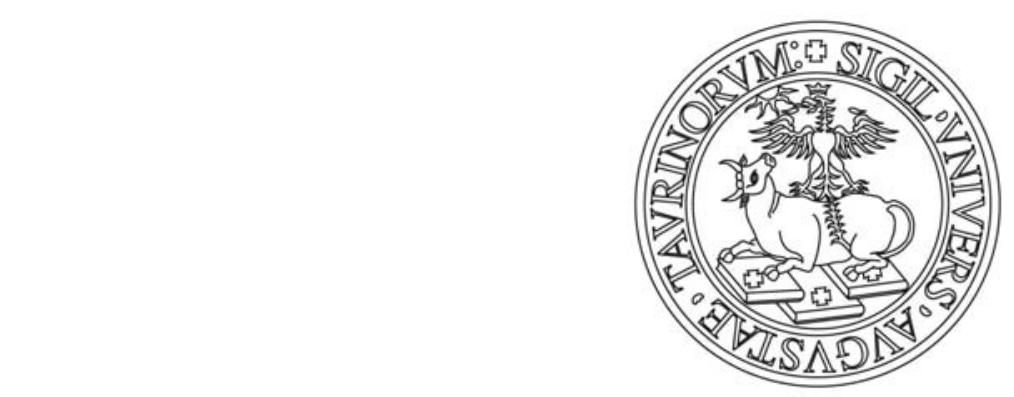

\section{UNIVERSITÀ DEGLI STUDI DI TORINO}

This is an author version of the contribution published on:

Questa è la versione dell'autore dell'opera:

Cavallo A., Bucchioni G., Castiello U., Becchio C. (2013). Goal or movement? Action representation within the primary motor cortex. European Journal of Neuroscience, 38, 3507-3512.

The definitive version is available at:

La versione definitiva è disponibile alla URL:

http://onlinelibrary.wiley.com/doi/10.1111/ejn.12343/abstract;jsessionid=4844D8E 71D95A1AD60281A852DCD4A62.f04t03 
Journal section: Research reports

\title{
Goal or movement?
}

\section{Action representation within the primary motor cortex}

\author{
Andrea Cavallo $^{1}$, Giulia Bucchioni ${ }^{2}$, Umberto Castiello ${ }^{3}$, Cristina Becchio ${ }^{1 *}$
}

${ }^{1}$ Centre for Cognitive Science, Department of Psychology, University of Torino, Torino, Italy

${ }^{2}$ Laboratoire de Neuroscience Functionelles et Pathologies, UFR de Médicine, Université de Picardie Jules Verne, Amiens, France

${ }^{3}$ Department of General Psychology, University of Padova, Padova, Italy

* correspondence to: Cristina Becchio, Department of Psychology, University of Turin; Via Po, 14, 10123 Turin, Italy; Fax: +39 011 8146231; E-mail address: cristina.becchio@unito.it

Running title: Goal or movement?

Page counts: 16

Figure counts: 2

Word counts: 4921 


\begin{abstract}
Although facilitation of the corticospinal system during action observation is widely accepted, it remains controversial whether this facilitation reflects a replica of the observed movements or the goal of the observed motor acts. In the present TMS study we recorded motor evoked potentials (MEPs) from two hand muscles (first dorsal interosseous, FDI; abductor digiti minimi, ADM) while 22 healthy participants observed a hand reaching towards and grasping a bottle. To test for alternative coding levels (goal versus movement), three relevant aspects were systematically manipulated: the type of observed movement (precision grip or whole hand grasping), the situational context (bottle positioned in front or behind a wall-like barrier), and processing stage (TMS pulse delivered at the onset of the movement or at the moment of contact between the fingers and the object). At movement onset, MEP responses reflected the program necessary to achieve the action goal within the situational context. During movement observation, however, the type of observed movement was taken into account and a transition towards a movement-related modulation was observed. These results suggest that rather than being exclusive alternatives, goal coding and movement coding may relate to different processing stages.
\end{abstract}

Keywords: transcranial magnetic stimulation; action observation; motor facilitation; reach to grasp movement; goal coding 


\section{Introduction}

Observation of other people's actions has been shown to selectively facilitate the brain's motor circuits for executing the same actions (Grafton, 2009; Rizzolatti \& Sinigaglia, 2010). But how exactly are observed actions mapped on the observer's motor system? The same motor behavior can be described at different levels: the goal level, the kinematics of the executed movement, the specific motor commands that activate the muscles in a coordinated sequence (Grafton \& Hamilton, 2007). Which level of the hierarchy is relevant for motor facilitation? When a goal is present, is the pattern of muscle recruitment linked to the observed movements or to the goal of the observed motor act?

Action goals have been show to influence response properties across different levels of the parieto-frontal network for action observation (for review see Grafton, 2009; Grafton, 2010; Rizzolatti \& Sinigaglia, 2010). Within the primary motor cortex, sensitivity to action goals is suggested by the finding that TMS-induced motor evoked potentials (MEPs) vary depending on whether a goal is present or not (Cattaneo et al., 2009). When there was no goal in the observed behavior (i.e., the experimenter merely opened and closed classic or reverse pliers), MEPs reflected the movements performed by the agent. However, when a goal was present (i.e., the experiment grasped and dropped a peanut using the classic or reverse pliers), MEPs no longer reflected the observed movements, but the movements necessary to achieve the goal, suggesting that observed movements were remapped on the same goal-directed motor act. Others studies seeking to dissociate goal and movement, however, failed to show goal-related modulation of M1 excitability, revealing instead a faithful replica of the observed movement (Borroni et al., 2011; Cavallo et al., 2012; Sartori et al., 2012). For example, Cavallo et al. (2012) found that MEPs recorded from hand muscles during observation of tool actions reflected the observed hand movement, rather than the movement of the tool or the distal goal of the action. Therefore it remains an open question whether the excitability of M1 modulates according to the goal or the observed movements. 
To elucidate the specificity of the visuo-motor response, Lago and Fernandez-del-Olmo (2011) recorded MEPs from hand muscles during observation of grasping actions performed with the hand or the foot. When participants observed a static effector in front of the object, MEP enhancement was similar for observation of hand and foot actions. During the observation of the effector-object interaction, however, the initial facilitation observed for foot actions was abolished, suggesting a transition towards a more specific movement-related modulation of MEP amplitude.

In this perspective, one possibility is that the properties of motor responses to observed actions differ according to the processing stage. Before the to-be-observed action starts, contextual factors might dictate a motor facilitation reflecting the motor program necessary to achieve an action goal. During movement observation, computation of the specific features of the movement would then override the initial goal representation, providing a refined matching of the unfolding movement. If this is correct, then one would expect a reversal in muscle-specific MEP enhancement when the observed movement does not correspond to the motor program initially facilitated.

In the present study we explored this prediction using a novel paradigm adapted from infant research. In violation-of-expectation studies, goal-directed actions are demonstrated to infants within different situational constraints in order to test for alternative encoding of unusual actions (Gergely \& Csibra, 2009; Philips \& Wellman, 2005). By following the same logic, in our study we varied the presentation of different types of actions movements and situational constraints to examine the modulation of M1 excitability by goal and movement. Participants observed a hand reaching towards and grasp a bottle positioned in front or behind a wall-like barrier in three conditions. For the 'precision grip constrained' (PG_constrained) condition, the bottle was placed behind the barrier, so that the barrier prevented the direct reach of the bottle. Participants observed a model's hand reaching over the barrier and grasping the bottle using a precision grip (Figure 1a). For the 'precision grip unconstrained' (PG_unconstrained) condition, participants observed the model's hand grasping the bottle, located in front of the barrier, with a precision grip (Figure 1b). Finally, there was a 'whole hand grasp' (WHG) condition in which participants observed the 
model's hand grasping the bottle, placed in front of the barrier, with a whole hand grasp (Figure 1c). We expected that MEPs responses recorded at the time the observed movement started should be similar for the PG_unconstrained and the WHG conditions. This is because the situational context (bottle in front the barrier) should facilitate the same goal-directed motor program for both conditions. During movement unfolding, however, a transition towards a more specific movementrelated modulation of MEP amplitude should be observed. If this is the case, then, at the time the hand enters in contact with the object, MEP responses for the PG_unconstrained condition would be expected to reverse as to match the specific features of the observed movement. This should not occur for the WHG and the PG_constrained condition, in which the movement unfolds in accordance with the initially facilitated motor program.

\section{Materials and methods}

\section{Participants}

Twenty-two healthy volunteers (7 men and 15 women) aged 20-36 (mean 24.3 years) took part in the experiment. All were right-handed, had normal or corrected-to-normal visual acuity and were free from any contraindication to TMS (Wassermann, 1998; Rossi et al., 2009). None of them had a history of neurological, major medical, or psychiatric disorders. The experimental procedures were approved by the ethical committee of the University of Padova and were carried out in accordance with the principles of the revised Helsinki Declaration (World Medical Associations General Assembly, 2008). Written informed consent was obtained from each subject prior to experimentation. None of the individuals taking part in the experiment experienced discomfort or adverse effects during TMS acquisitions. Experiments were conducted in accordance with the ethical guidelines

\section{Stimuli}


The experimental stimuli consisted of videoclips representing three types of action sequences in which a human right hand reached towards, grasped, and lift a bottle positioned in front of or behind a wall-like barrier. For the 'Precision grip constrained' condition (PG_constrained) the bottle was placed behind the barrier. The model's hand reached over the barrier and grasped the bottle by the cap with a precision grip, i.e., by opposing the thumb with the index finger (Figure 1a). For the 'Precision grip unconstrained' condition (PG_unconstrained), although the bottle was in front of the barrier, the model's hand moved in the same manner as for the PG_constrained condition grasping the bottle by the cap with a precision grip (Figure 1b). For the 'Whole hand grasp' condition (WHG) the hand reached, grasped and lifted the bottle positioned in front of the barrier using a whole-hand grasp, i.e., by using the thumb and hand palm (Figure 1c). At the beginning of each sequence, the hand of the model was shown resting on a table in a prone position. The reaching movements started $500 \mathrm{~ms}$ after the onset of the action sequence. Video clips in which the hands moved towards the bottle but no hand-object interaction was displayed were also included as 'catch' trials. Each video clip lasted $4000 \mathrm{~ms}$ and the animation effect was obtained by presenting series of single frames each lasting $33 \mathrm{~ms}$ except for the first and last frame which lasted 500 and $893 \mathrm{~ms}$, respectively.

\section{Electromyographic and TMS Recording}

When agents shape their hand to grasp an object there is a characteristic pattern of muscle activity in the intrinsic muscles of the hand that move the index (First Dorsal Interosseous, FDI) and little finger (Abductor Digiti Minimi, ADM; e.g., Cattaneo et al., 2005). The FDI muscle abducts and flexes the index finger and is involved in precision grip (PG) and, to a lesser extent, in whole hand grasp (WHG); in contrast, the ADM muscle abducts the little finger and is significantly more active for WHG than PG. Because of this characteristic pattern of activation, FDI and ADM are commonly considered when evaluating muscle-specific MEP enhancement during observation of PG and WHG actions (e.g., Fourkas et al., 2006; Urgesi et al., 2010; Sartori et al., 2012). 
Following this logic, in the present study we recorded MEPs from FDI and ADM. TMS pulses were administered by using the Master Magstim 200 Unit of a Magstim Bistim2 stimulator (Magstim, Whitlan, Dyfed, Wales, UK) connected to a $70 \mathrm{~mm}$ figure-of-eight coil. The coil was held tangentially to the scalp with the handle pointing backwards and laterally with a $45^{\circ}$ angle to the midline. This orientation permits to achieve the lowest motor threshold, optimizing the stimulation (Brasil-Neto et al. 1992; Mills et al. 1992). During the recording session, the coil was positioned in correspondence with the optimal scalp position (OSP), defined as the position from which Motor Evoked Potentials (MEPs) with maximal amplitude were recorded simultaneously from FDI and ADM. To find individual OSP, the coil was moved in steps of $1 \mathrm{~cm}$ over the motor cortex and the OSP was marked on a bathing cap worn by the participants. Once the OSP was found, the individual resting motor threshold (rMT) was determined as the lowest stimulus intensity able to generate reliable MEPs ( $\geq 50 \mu \mathrm{V}$ peak-to-peak amplitude) in the relaxed muscles in 5 out of 10 consecutive TMS pulses (Rossini et al. 1994). During the recording sessions, stimulation intensity was set at $115 \%$ of the rMT which ranged from $38 \%$ to $62 \%$ (mean $47.14 \%$ ) of the maximum stimulator intensity and the OSP was continuously checked during the whole experiment. MEPs were simultaneously recorded from FDI and ADM muscles of the participants' right hand. Electromyographic (EMG) activity was recorded by pairs of $\mathrm{Ag}-\mathrm{AgCl}$ surface electrodes $(9 \mathrm{~mm}$ diameter) placed over the muscle belly (active electrode) and over the ipsilateral proximal interphalangeal joint (reference electrode) in a classical belly-tendon montage. Electrodes were connected to an isolated portable ExG input box linked to the main EMG amplifier for signal transmission via twin fiber optic cable (Professional BrainAmp ExG MR, Brain Products, Munich, Germany). The ground electrode was placed over the participants' left wrist and was connected to the common input of the ExG input box. In the recording session EMG signals were sampled (5000 $\mathrm{Hz})$, amplified, band-pass filtered $(20 \mathrm{~Hz}-1 \mathrm{kHz})$, and stored on a PC for off-line analysis. A prestimulus recording of $100 \mathrm{~ms}$ was used to check for the presence of EMG activity before the TMS pulse. In order to prevent contamination of MEP measurements by background EMG activity, 
trials with any background activity greater than $100 \mu \mathrm{V}$ in the $100 \mathrm{~ms}$ window preceding the TMS pulse were excluded from the MEP analysis. EMG data were collected for $300 \mathrm{~ms}$ after the TMS pulse.

\section{Procedure}

Participants were tested in a single experimental session lasting approximately 45 minutes. Experimentation was carried out in a dimly illuminated room. Participants were seated in a comfortable armchair with a fixed chinrest. Stimuli were displayed on a 19-inch monitor (resolution $1280 \times 1024$ pixels, refresh frequency, $75 \mathrm{~Hz}$ ) at a viewing distance of $80 \mathrm{~cm}$. Participants were instructed to pay attention to the displayed stimuli while keeping their right hand still and as relaxed as possible. As a control for attention, they were told that at the end of the experiment they would be debriefed about what they had seen during the experimental session. The magnetic pulse was delivered at the onset of the movement - when the hand started the action ('start') - or at the moment of contact between the fingers and the object ('contact'). During the TMS session eight trials were presented for each type of action sequence (PG_constrained, PG_unconstrained, WHG) for each pulse delay (start, contact). 16 additional catch trials ( 8 for start and 8 for contact delay) were shown for a total of 64 randomly presented trials. Two series of 5 MEPs were also recorded while participants were observing a white fixation cross presented on a black background. One series was recorded at the beginning, whereas the second was recorded at the end of the experimental session. Comparisons of MEP amplitudes for the two series allowed us to check for any corticospinal excitability change related to TMS per se. Following each action sequence, an inter trial interval of $9 \mathrm{~s}$ was given: a message asking participants to keep their hand still and fully relaxed was presented during the first $7 \mathrm{~s}$ and then replaced by a fixation cross for the remaining $2 \mathrm{~s}$. Stimulus-presentation timing, EMG recording and TMS triggering, as well as randomization of stimuli were controlled by using E-Prime V2.0 software (Psychology Software Tools Inc., Pittsburgh, PA, USA) running on a PC. 


\section{Results and statistical analyses}

\section{Data analysis}

Data were analyzed off-line using Brain Vision Analizer software (Brain Products GmbH, Munich, Germany) and SPSS 17.0.1 (SPSS Inc., Chicago, IL, USA). Background EMG level prior to TMS was calculated for each trial. Individual mean peak-to-peak amplitudes of MEPs recorded from the FDI and ADM muscles were calculated separately for each type of action sequence (PG_constrained, PG_unconstrained, WHG) and TMS pulse delay (start, contact). MEP amplitudes and onset latencies deviating more than 2 standard deviations (SD) from the mean of each experimental condition and single trials contaminated by muscular preactivation were excluded as outliers and precontracted trials respectively. The resulting average number of MEPs in any cell for each participant was $7.29 \pm 0.50$. The individual mean amplitude of MEPs recorded from both muscles during fixation cross trials (10 out of 74 trials) served as baseline for MEPs of trials where movements were shown, so that MEP amplitudes were converted into a proportion of the baseline value. From converted data, the MEP ratio was calculated for each trial by dividing the ADM data point by the FDI data point. The resulting MEP ratio was then normalised using log10 transformation to address non-normality resulting from positive skew (Osborne, 2002). MEP ratio is considered to reflect the effectiveness of muscle specific mapping of the observed movement onto the motor system (Catmur, et al., 2010). Values of this index greater than zero indicate that ADM was more strongly involved than FDI, as expected during whole hand prehension; values lower than zero indicate that FDI was more strongly involved than ADM, as expected during precision grip.

Normalised data were submitted to a $3 \times 2$ repeated-measures analyses of variance (ANOVA) with type of action sequence (PG_constrained, PG_unconstrained, WHG), and TMS pulse delay (start, contact) as within-subjects factors. A paired sample t-test (2-tailed) was used to compare the amplitude of MEPs collected from the two muscles in the baseline trials. Finally, to control for 
differences in MEP onset latencies across conditions, onset latencies were submitted to a $3 \times 2 \times 2$ repeated-measures ANOVA with type of observed movement (PG_constrained, PG_unconstrained, WHG), TMS pulse delay (start, contact), and muscle (FDI, ADM) as within-subjects factors. A significance threshold of $\mathrm{P}<.05$ was set for all statistical tests.

\section{Results}

Mean raw MEP amplitudes during the two baseline blocks ran at the beginning and at the end of the experimental session were not significantly different for either the FDI $\left(t_{21}=.970, p=.343\right)$ or the ADM muscle $\left(t_{21}=1.417, \mathrm{p}=.171\right)$. This indicates that, in our experimental procedure, TMS per se did not induce any changes in corticospinal excitability. The $3 \times 2$ ANOVA on normalised MEP ratios yielded a statistically significant main effect of type of action sequence $\left[\mathrm{F}_{(1,21)}=10.832\right.$, $\mathrm{p}<.01]$ indicating a linear trend in the pattern of modulation. In particular, MEP ratios were greater for the WHG condition $(\mathrm{M}=.062)$, lower for the $\mathrm{PG}$ - unconstrained condition $(\mathrm{M}=.001)$, and lowest for the PG_constrained condition ( $\mathrm{M}=$-.019). Post hoc comparisons confirmed that MEP ratios were greater for the WHG than for the PG_constrained condition $(p=.003)$. In contrast, no statistical difference was found comparing the WHG and PG_unconstrained $(p=.110)$ and the PG_constrained and the PG_unconstrained condition $(\mathrm{p}=.544)$. This may reflect different mechanisms: First, it is possible that sensitivity to situational constraints modulated muscle specific mapping during movement observation. Second, it might be that, in line with our experimental hypothesis, the mapping of the observed movement onto the observer's motor system differed depending on the processing stage such that goal-related modulation to situational constraints at movement onset turns into movement-related modulation during movement observation. To explore this possibility we compared MEPs ratios at start and contact for each type of action sequence.

Paired t-tests performed separately for each type of action confirmed the hypothesis of a stage specific modulation. As illustrated in Figure 2, the comparison between the start and the contact time yielded a statistically significant effect for the PG_unconstrained condition $\left(\mathrm{t}_{21}=\right.$ 
$2.258, p=.035)$ suggesting a reversal in MEP responses. As predicted, the difference between time pulse delays (start, contact) for the PG_constrained and the WHG conditions was not significant $\left(\mathrm{t}_{21}\right.$ $=-.369, \mathrm{p}=.716 ; \mathrm{t}_{21}=-.662, \mathrm{p}=.515$, respectively). $3 \times 2 \times 2$ ANOVA on MEP onset latencies revealed no significant difference ( $\mathrm{p}_{\mathrm{s}}$ ranging from .153 to .993).

\section{Discussion}

Motor facilitation during action observation requires that the observed action is mapped, at some level of the action hierarchy, onto the observer's motor system. In the present TMS study we sought to investigate the level at which this visuo-motor mapping occurs within M1. Participants observed a hand reach towards and grasp a bottle. To test for alternative coding levels (goal versus movement) three relevant aspects were varied: the type of observed movement (precision grip or whole hand grasping), the situational context (bottle positioned in front or behind a wall-like barrier), and processing stage (TMS pulse delivered at the onset of the movement or at the moment of contact between the fingers and the object).

The main result of our experiment was the demonstration of a transition from goal-related modulation towards movement-related modulation when the observed movement did not correspond to the motor program initially facilitated (PG_unconstarined condition). At movement onset, MEP responses in the PG_unconstrained condition were similar to the WHG condition. This suggests that at movement start, facilitatory modulation reflected the program necessary to achieve the action goal within the situational context. When no physical constraint barred a direct reach of the bottle (PG_unconstrained and WHG conditions), observers displayed a muscle facilitation pattern compatible with a whole hand grasp. When the bottle was placed behind the barrier and a direct reach of the bottle was therefore prevented (PG_constrained condition), observer displayed a facilitation pattern compatible with a precision grip.

In the WHG and in the PG_constrained conditions the action unfolded in accordance with the initially facilitated motor program. Consistently, no significant difference was observed in MEP 
ratios between the start and the contact phase. In the PG_unconstrained condition, in contrast, although no physical constraint prevented a direct reach of the bottle, the model's hand grasped the bottle with a precision grip. As predicted, this unexpected unfolding determined a reversal in MEP responses at the time the hand entered in contact with the object, suggesting that the initial motor program (whole hand grasp) was substituted with a new plan taking into account the specific features of the observed movement (precision grip).

Motor facilitation during action observation has been proposed to result from the comprehensive loading of a motor plan at the beginning of action observation (Gangitano et al., 2004). Our findings extend this literature by suggesting that the goal-related motor plan loaded before movement onset can be substituted by a new plan as to match the specific properties of the observed movement. In this manner, the expectation of a predictable ensuing movement may be integrated with the modulation triggered by the observation of the actual movement.

One limitation of the present design is that it does not allow characterizing the time-course of the MEP transition from goal-coding to movement coding, i.e., when during action unfolding MEP modulation ceases to reflect the goal of the action to reproduce the actual observed movement. Gangitano et al., (2004) found that unpredictable unnatural features (sudden closure movement of the hand) inserted $1200 \mathrm{~ms}$ after grasping onset exerted no cortico-spinal modulation in the motor system of the observer. This may indicate that the substitution of the initially facilitated motor plan can take place immediately after the observed movement onset, but not during movement unfolding. However, it is also possible that integration is limited to features matching a 'natural motor template' (Gangitano et al., 2004). The resonant plan evoked at the beginning of the movement may be substituted by a new plan during movement unfolding, but only when the unexpected features match the motor plans resident in the observer motor system. Future investigations could use additional stimulation time-points between action onset and completion to clarify whether plan substitution can take place in flight in response to natural changes in the observed movement and to characterize the exact time-course of this substitution. 
It may be argued that reversal in MEP responses for the PG_unconstrained condition reflects the decay of the initial activation rather than the loading of a new motor plan. According to this alternative explanation, the initial motor plan neither takes into account the unexpected features of the observed movement nor is substituted by a new plan. Once activated, it loads the features of the predictable ensuing movement and is discarded when these features cease to match the visual properties of the observed movement (Gangitano et al. 2004). In such case, however, finger closure in the PG_unconstrained condition should not exert any modulatory effect. Moreover, a significant difference should be expected between MEPs ratios for the PG_unconstrained condition and the PG_constrained condition at contact. This was not the case $\left(\mathrm{t}_{21}=.744, p=.448\right)$. These findings suggest that the unexpected unfolding was substituted by a new plan rather than by a slow decay of the initial modulation. Future research employing additional stimulation time-points delivered at the time of appearance of specific kinematic features (e.g., finger opening) may help to examine this alternative hypothesis more closely. If motor output modulation is the result of deployment of the original plan, then there should be disparity between MEP modulation and kinematic profiles (Gangitano et al., 2004). If, on the contrary, reversal in MEP responses results from the loading of a new plan, then the pattern of corticospinal excitability should follow the dynamics of the observed movements.

To date, the action level relevant for visuo-motor mapping in M1 is still debated (Borroni et al., 2011; Cattaneo et al., 2009; Cavallo et al., 2012; Sartori et al., 2012). The present findings provide a means to integrate contrasting perspective on goal and movement coding. In particular, they suggest that, rather being mutually exclusive alternatives, modulation to goal and movement may relate to different processing stages. Goal coding may take place at movement onset. At this stage, motor excitability is modulated according to situational constraints and, regardless of the observed effector, reflects the muscle facilitation pattern necessary to achieve the goal of the action (Lago \& Fernandez-del-Olmo, 2011). During movement observation, however, the type of movement and the effector are taken into account and a transition towards movement-related 
modulation is observed (Alaerts et al., 2009; Aziz-Zadeh et al. 2002; Fadiga et al., 1995; Gangitano et al. 2001; Maeda et al. 2002; Montagna et al. 2005; Strafella \& Paus 2000; Urgesi et al. 2006; Urgesi et al. 2010). The goal-directed program initially facilitated by the observation of a static effector is in this way translated into a program representing the specific features of the observed movement.

\section{Acknowledgments}

This work was supported by a grant from the Regione Piemonte, bando Scienze Umane e Sociali 2008, L.R. n.4/2006.

Abbreviations: TMS, transcranial magnetic stimulation; M1, primary motor cortex; MEP, motor evoked potential; WHG, whole hand grasp; PG_constrained, precision grip constrained; PG_unconstrained, precision grip unconstrained; FDI, first dorsal interosseous; ADM, abductor digiti minimi 


\section{References}

Alaerts, K., Heremans, E., Swinnen, S.P. \& Wenderoth, N. (2009) How are observed actions mapped to the observer's motor system? Influence of posture and perspective. Neuropsychologia, 47, 415-422.

Aziz-Zadeh, L., Maeda, F., Zaidel, E., Mazziotta, J. \& Iacoboni, M. (2002) Lateralization in motor facilitation during action observation: a TMS study. Exp. Brain. Res., 144, 127-131.

Borroni, P., Gorini, A., Riva, G., Bouchard, S. \& Cerri, G. (2011) Mirroring avatars: dissociation of action and intention in human motor resonance. Eur. J. Neurosci., 34, 662-669.

Brasil-Neto, J.P., Cohen, L.G., Panizza, M., Nilsson, J., Roth, B.J. \& Hallett, M. (1992) Optimal focal transcranial magnetic activation of the human motor cortex: effects of coil orientation, shape of the induced current pulse, and stimulus intensity. J. Clin. Neurophysiol., 9, 132-136.

Cattaneo, L., Voss, M., Brochier, T., Prabhu, G., Wolpert, D. M. \& Lemon, R. N. (2005). A corticocortical mechanism mediating object-driven grasp in humans. Proc. Natl. Acad. Sci. U S A, 102, 898-903.

Cattaneo, L., Caruana, F., Jezzini, A. \& Rizzolatti, G. (2009) Representation of goal and movements without overt motor behavior in the human motor cortex: a transcranial magnetic stimulation study. J. Neurosci., 29, 11134-11138.

Cavallo, A., Becchio, C., Sartori, L., Bucchioni, G. \& Castiello, U. (2012) Grasping with tools: corticospinal excitability reflects observed hand movements. Cereb. Cortex., 22, 710-716.

Csibra, G. \& Gergely, G. (2009) Natural pedagogy. Trends Cogn. Sci., 13, 148-153.

Fadiga, L., Fogassi, L., Pavesi, G. \& Rizzolatti, G. (1995) Motor facilitation during action observation: a magnetic stimulation study. J. Neurophysiol., 73, 2608-2611.

Fourkas, A. D., Avenanti, A., Urgesi, C. \& Aglioti, S. M. (2006). Corticospinal facilitation during first and third person imagery. Exp. Brain Res., 168, 143-151.

Gangitano, M., Mottaghy, F.M. \& Pascual-Leone, A. (2001) Phase-specific modulation of cortical motor output during movement observation. Neuroreport, 12, 1489-1492. 
Gangitano, M., Mottaghy, F.M. \& Pascual-Leone, A. (2004) Modulation of premotor mirror neuron activity during observation of unpredictable grasping movements. Eur. J. Neurosci., 20, $2193-$ 2202.

Grafton, S.T. (2009) Embodied cognition and the simulation of action to understand others. Ann. N. Y. Acad. Sci., 1156, 97-117.

Grafton, S.T. (2010) The cognitive neuroscience of prehension: recent developments. Exp. Brain Res., 204, 475-491

Grafton, S.T. \& Hamilton, A.F. (2007) Evidence for a distributed hierarchy of action representation in the brain. Hum. Mov. Sci., 26, 590-616.

Lago, A. \& Fernandez-del-Olmo, M. (2011) Movement observation specifies motor programs activated by the action observed objective. Neurosci. Lett., 493, 102-106.

Maeda, F., Kleiner-Fisman, G. \& Pascual-Leone, A. (2002) Motor facilitation while observing hand actions: specificity of the effect and role of observer's orientation. J. Neurophysiol., 87, 13291335.

Mills, K.R., Boniface, S.J. \& Schubert, M. (1992) Magnetic brain stimulation with a double coil: the importance of coil orientation. Electroencephalogr. Clin. Neurophysiol., 85, 17-21.

Montagna, M., Cerri, G., Borroni, P. \& Baldissera, F. (2005) Excitability changes in human corticospinal projections to muscles moving hand and fingers while viewing a reaching and grasping action. Eur. J. Neurosci., 22, 1513-1520.

Osborne J. (2002) Notes on the use of data transformation. Pract. Assess. Res. Eval., 8, http://PAREonline.net/getvn.asp?v $=8 \& n=6$

Phillips, A.T. \& Wellman, H.M. (2005) Infants' understanding of object-directed action. Cognition, 98, 137-155.

Rizzolatti, G. \& Sinigaglia, C. The functional role of the parieto-frontal mirror circuit: interpretations and misinterpretations. Nat. Rev. Neurosci., 11, 264-274. 
Rossi, S., Hallett, M., Rossini, P.M. \& Pascual-Leone, A. (2009) Safety, ethical considerations, and application guidelines for the use of transcranial magnetic stimulation in clinical practice and research. Clin. Neurophysiol., 120, 2008-2039.

Rossini, P.M., Barker, A.T., Berardelli, A., Caramia, M.D., Caruso, G., Cracco, R.Q., Dimitrijevic, M.R., Hallett, M., Katayama, Y., Lucking, C.H. \& et al. (1994) Non-invasive electrical and magnetic stimulation of the brain, spinal cord and roots: basic principles and procedures for routine clinical application. Report of an IFCN committee. Electroencephalogr. Clin. Neurophysiol., 91, 79-92.

Sartori, L., Cavallo, A., Bucchioni, G. \& Castiello, U. (2012) From simulation to reciprocity: the case of complementary actions. Soc. Neurosci., 7, 146-158.

Sartori, L., Xompero, F., Bucchioni, G. \& Castiello, U. (2012). The transfer of motor functional strategies via action observation. Biol. Lett., 8, 193-196.

Strafella, A.P. \& Paus, T. (2000) Modulation of cortical excitability during action observation: a transcranial magnetic stimulation study. Neuroreport, 11, 2289-2292.

Urgesi, C., Candidi, M., Fabbro, F., Romani, M. \& Aglioti, S.M. (2006) Motor facilitation during action observation: topographic mapping of the target muscle and influence of the onlooker's posture. Eur. J. Neurosci., 23, 2522-2530.

Urgesi, C., Maieron, M., Avenanti, A., Tidoni, E., Fabbro, F. \& Aglioti, S.M. (2010) Simulating the Future of Actions in the Human Corticospinal System. Cereb. Cortex, 20, 2511-2521.

Wassermann, E.M. (1998) Risk and safety of repetitive transcranial magnetic stimulation: report and suggested guidelines from the International Workshop on the Safety of Repetitive Transcranial Magnetic Stimulation, June 5-7, 1996. Electroencephalogr. Clin. Neurophysiol., 108, $1-16$.

World Medical Association General Assembly (2008) Declaration of Helsinki. Ethical principles for medical research involving human subjects. World Medical Journal, 54, 122-5. 


\section{Figure legends}

Figure 1: Schematic representation of event sequencing during PG_constrained (A),

PG_unconstrained (B) and whole hand grasp (C) actions. The TMS pulse could be delivered at the onset of the movement - when the hand started the action ('Start') - or at the moment of contact between the fingers and the object ('Contact').

Figure 2: Peak-to-peak amplitude scores recorded during the observation of different types of movement. MEPs amplitudes are expressed as a ratio calculated for each trial by dividing the ADM data point by the FDI data point. The resulting MEP ratio is normalised using log10 transformation. The dotted line indicates a significant linear trend in the pattern of modulation with MEP ratios being greater for the WHG condition, lower for the PG_unconstrained condition, and lowest for the PG_constrained condition. Asterisks indicate significant paired t-test comparisons $(p<0.05)$. 
Figure 1

Start
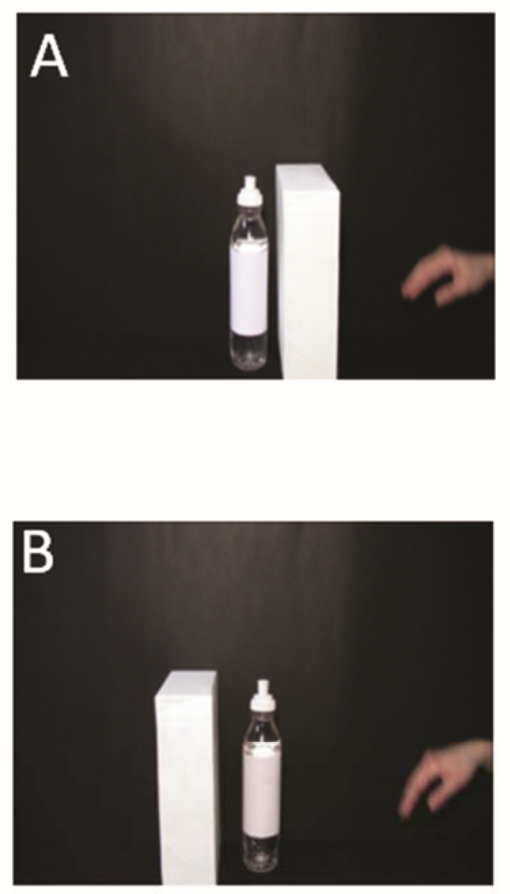

C
Contact
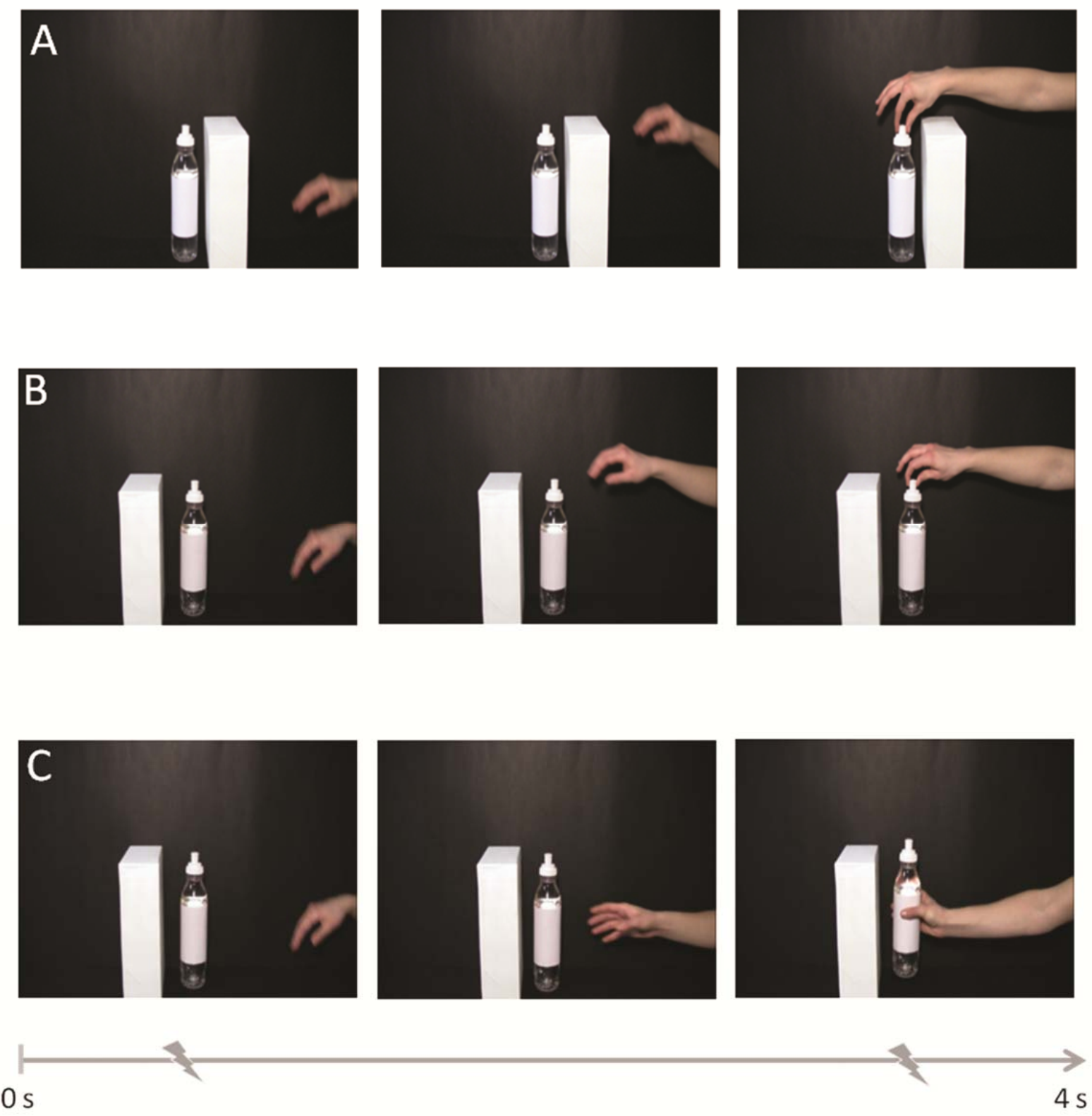
Figure 2

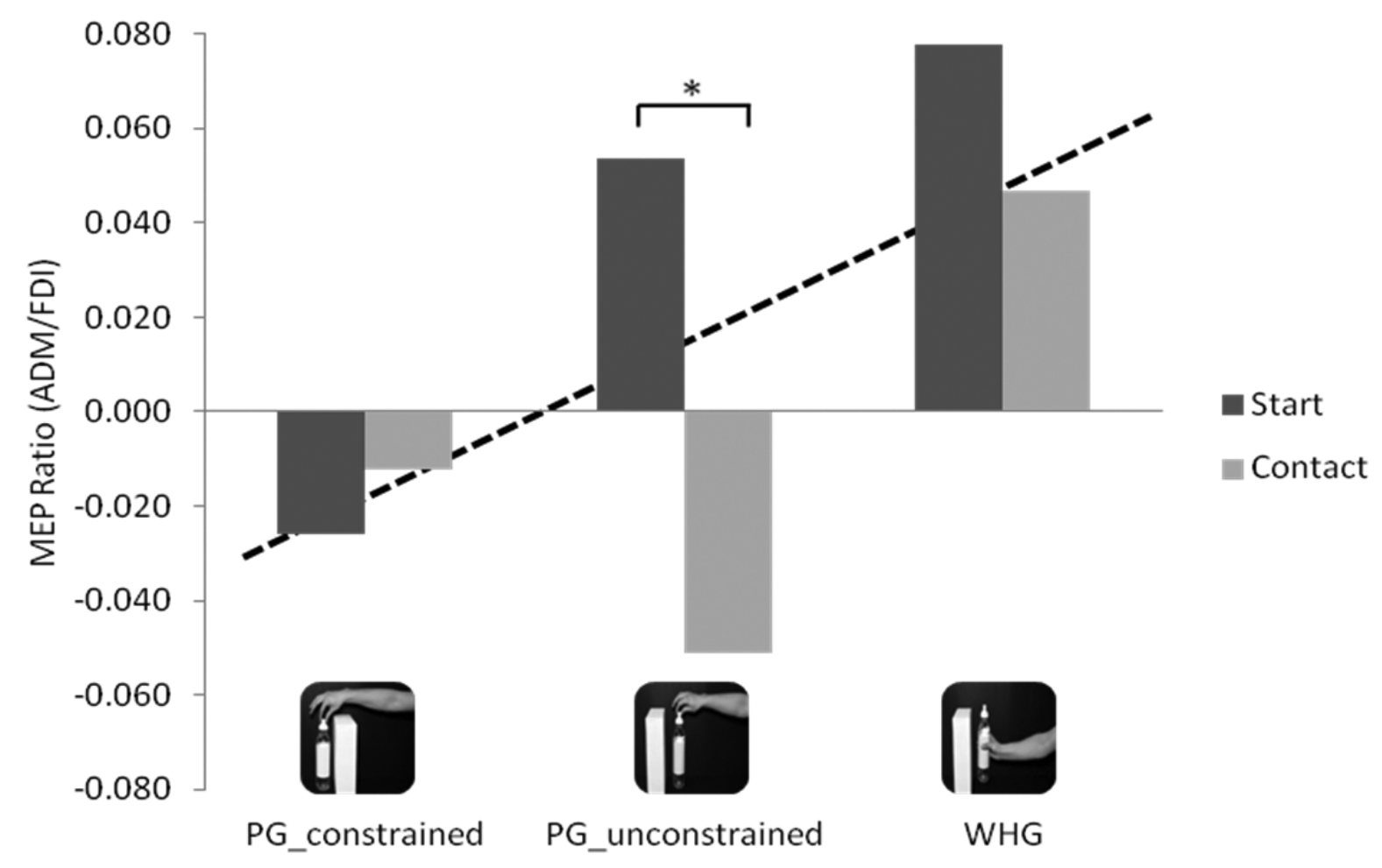

